\title{
Construction of Block-coil High-field Model Dipoles for Future Hadron Colliders
}

\author{
Raymond Blackburn, Tim Elliott, William Henchel, Al McInturff, Peter McIntyre, and Akhdior Sattarov
}

\begin{abstract}
A family of high-field dipoles is being developed at Texas A\&M University, as part of the program to improve the cost-effectiveness of superconducting magnet technology for future hadron colliders. The TAMU technology employs stress management, flux-plate control of persistent-current multipoles, conductor optimization using mixed-strand cable, and metalfilled bladders to provide pre-load and surface compliance. Construction details and status of the latest model dipole will be presented.
\end{abstract}

Index Terms - high-field dipoles, $\mathrm{Nb}_{3} \mathrm{Sn}$, stress management, collider magnets.

\section{INTRODUCTION}

$\mathrm{T}$ HE next generation of hadron colliders will require significant innovations in superconducting magnet technology to lower the cost per $\mathrm{TeV}$ of their systems. One obvious direction is to increase the magnetic field in the dipoles, beyond the planned LHC $\sim 8 \mathrm{~T}$, thus requiring an A-15 superconductor (SC). While $\mathrm{Nb}_{3} \mathrm{Sn}$ presently is the most mature A$15 \mathrm{SC}$, it poses challenges for both the fabrication and operation of the dipoles. First, $\mathrm{Nb}_{3} \mathrm{Sn}$ is brittle and windings are subject to strain degradation [1]. Second, Lorentz stress accumulates as one increases magnetic field strength $\left(\sim B^{2}\right)$, so that at sufficiently high fields a homogeneous winding could suffer strain degradation. Third, the diffusion reaction that forms the superconducting A15 phase in the SC strands of the cable is prone to bridging adjacent filaments. This produces a large effective filament size and correspondingly large persistent-current magnetization and therefore enhanced snap-back effects as well from boundary-induced current loops (BICCs)[2].

These challenges motivated a novel approach to the design and construction in SC dipole technology. The "wind and react" process having produced the highest operational current densities in high-field model dipoles, was chosen as the preferred fabrication mode [3].

- Stress management - The winding is configured in a block-coil geometry, and a high-strength support matrix is integrated within the coil so that the Lorentz stress devel-

Manuscript received August 6, 2002. This work was supported in part by the U.S. Department of Energy under Grant DE-FG03-95ER40924.

All authors are with the Department of Physics, Texas A\&M University, College Station, TX 77843 USA (phone: 979-845-6015; fax: 979-862-4730; e-mail: p-mcintyre@physics.tamu.edu). AM on leave from LBNL, Berkeley, CA. oped on the inner windings is intercepted and passed directly to the flux return and preload structure [4].

- Conductor optimization - The windings require sufficient volume of copper to carry current during a quench so that local heating is limited. The additional copper needed in the outer windings (where B is least and $\mathrm{j}_{\mathrm{c}}(\mathrm{SC})$ is greatest) is provided by cabling copper strands along with the composite $\left(\mathrm{Nb}_{3} \mathrm{Sn}\right.$ and copper) SC strands [5], or by cabling the composite SC strands around a copper/stainless steel core, thereby minimizing the volume of composite strand.

- Flux plate suppression of persistent-current magnetization - A horizontal flux plate of $(\mathrm{Fe})$ steel is inserted between the mid-plane and top/bottom windings. The steel is unsaturated at injection field and the initiation of acceleration. This close-coupled planar boundary condition provides a strong suppression of persistent-current fields and also of BICC's, reducing the calculated snap-back to one third of the previous conductor-dominated designs [6].

A NbTi block model dipole was constructed and tested in 2000, which contained many of the above design elements [7]. This paper reports the construction status of the first $\mathrm{Nb}_{3} \mathrm{Sn}$ block model dipole encompassing the above design elements and its current fabrication evolution. A discussion of a design that optimizes the above design criteria for the minimum aperture required for an ultimate-energy hadron collider is contained in 2001 Snowmass Conference Proceedings [8].

\section{12 Tesla Block Coil Dipole}

Fig. 1 shows the design of a $\mathrm{Nb}_{3} \mathrm{Sn}$ block-coil dipole embodying the above design elements. Its main parameters are given in Table I. Adopting the LBNL groups' modular coil approach [3], the block coil is configured in three subassemblies, each fabricated (winding, reaction bake, impregnation, and packaging) as a freestanding module. This has the advantage that the dipole can be built and tested in stages, first one module, then two together, and finally all three.

TABLE I

MAIN PARAMETERS OF 12 TESLA BLOCK-COIL DIPOLE

\begin{tabular}{lrl}
\hline \hline Central field, short sample limit & 13.2 & $\mathrm{~T}$ \\
Bore tube diameter & 30 & $\mathrm{~mm}$ \\
Coil current & 13 & $\mathrm{kA}$ \\
Coil cross section (superconducting strand) & 29 & $\mathrm{~cm}^{2}$ \\
Maximum Lorentz stress in coil & 108 & $\mathrm{MPa}$ \\
Inductance & 7.88 & $\mathrm{mH} / \mathrm{m}$ \\
Stored energy & 666. & $\mathrm{~kJ} / \mathrm{m}$ \\
\hline
\end{tabular}




\begin{tabular}{lrl}
\hline \hline Central field, short sample limit & 13.2 & $\mathrm{~T}$ \\
MIITS & 21 & $\mathrm{MA}^{2} \mathrm{~s}$ \\
\hline \hline
\end{tabular}
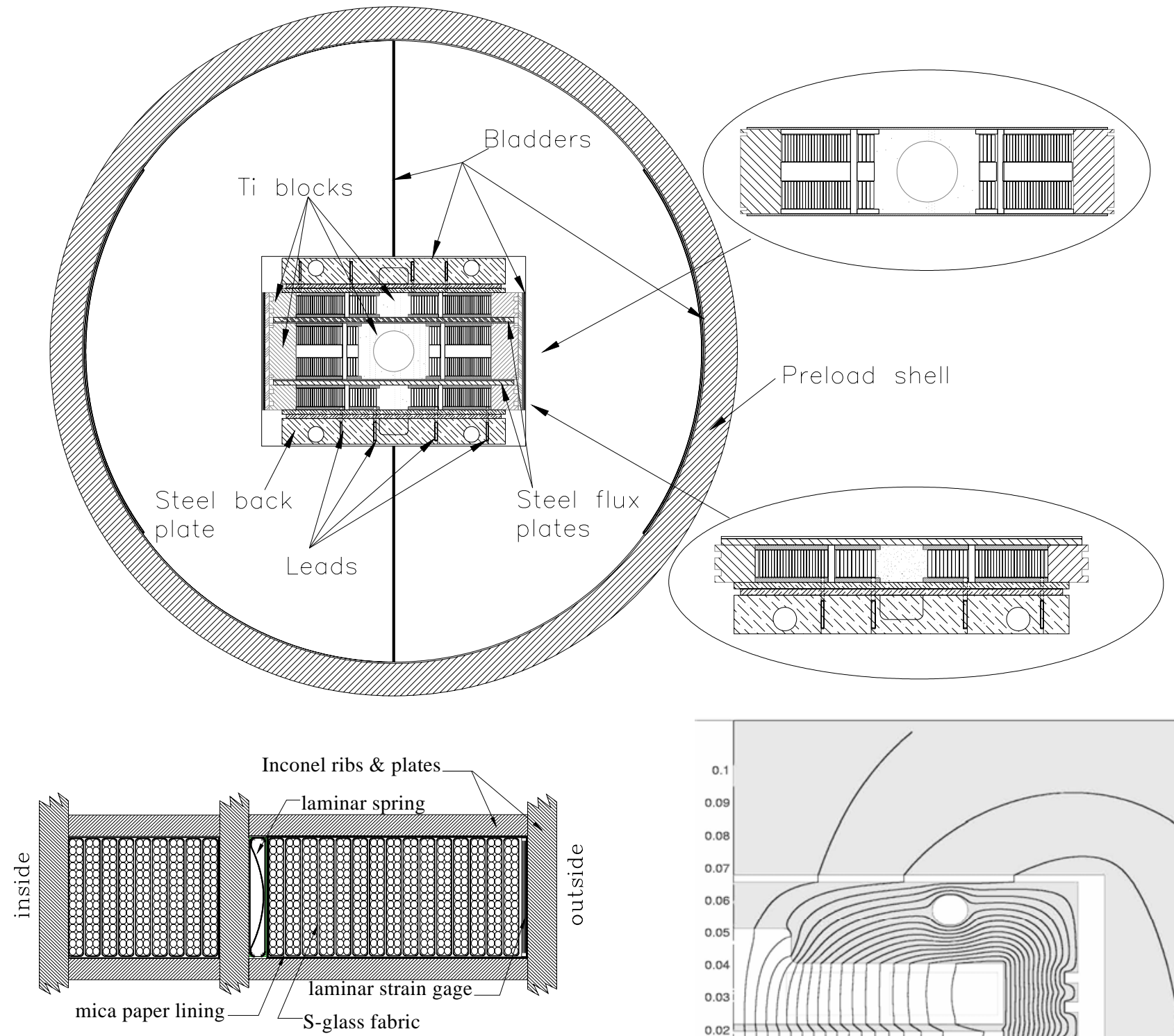

Fig. 1. 12 Tesla block-coil dipole, showing details of coil subassemblies, preload bladders, flux plates, and Ti blocks.

Fig. 2. Detail of two coil blocks showing elements of stress management.

The windings in each coil subassembly are divided into inner and outer windings. An Inconel support matrix is integrated into the coil, bypassing inner coil generated stress around the outer windings to the return shield.

\section{A. Stress management}

Stress management entails the integration of a high-strength support matrix within the coil, so that Lorentz stress can be intercepted and bypassed. This is accomplished via three key elements, shown in Fig. 2. The support matrix consists of a set of ribs and plates, made from Inconel 718. The parts are used with mill finish (no surface machining), and are cut from sheet using electric discharge machining. The laminar spring is formed from $200 \mu \mathrm{m}$ Inconel X750 foil, stamped to form the 
or less thus below the level for strain degradation of the $\mathrm{Nb}_{3} \mathrm{Sn}$ strand.

\section{B. Conductor Optimization}

The cables of the inner windings consist entirely of composite SC strands, containing a copper fraction Cu:total = 0.50 . The outer winding high performance cables contain half SC strands and half copper strands as discussed above, reducing their cost up to $40 \%$. For $\mathrm{Nb}_{3} \mathrm{Sn}$ cables, their cost is driven by the volume of the composite SC strand. This separated copper approach has an additional advantage of easily controlling the copper resistivity not being subject to contamination during reaction bake (e.g. bronzing due to tin leakage). There are still cabling issues unsolved and cable quench performance is not up to present standards. The first ITER strand outer module being fabricated, will not need the extra copper in the cable.

\section{Bladder pre-load}

The uniform pre-load to the coil assembly is delivered by the expansion bladders. The flux return is divided into two halves in the vertical mid-plane. The coil assembly surrounded by the bladders is inserted into a rectangular cavity of the flux return. The flux return with a pair of curved bladders outside each half is inserted in the aluminum shell. The entire assembly is then heated to $\sim 70 \mathrm{C}$, the bladders are evacuated, and then a low-melt alloy is pumped into the bladders under pressure to the desired pre-load. The bladder's thin stainless foil conforms to the finished surface of the coil assembly and flux return cavity resulting in a uniform pressure. By controlling the pressures both inside and outside independently, the pre-load is controlled on the entire assembly. Under pressure the magnet is then cooled to solidify the alloy. The preloaded magnet is ready to cool down and energize. A low-melt alloy was selected that has near-zero aggregate temperature contraction for the cycle $343 \rightarrow 4 \mathrm{~K}$. The alloy has moderate electrical conductivity, and a loss in resistance of three at $4 \mathrm{~K}$. A further virtue of bladder preload is that the dipole is easily disassembled, so that coil subassemblies can be re-used in subsequent larger assemblies of the model dipoles. Bladders fabricated using the present laser-welds and weld preparations have exceeded desired pressures five fold and doubled the expansions foreseen without failure.

\section{Titanium blocks}

In later models the ends of the center subassembly will be bent into $90^{\circ}$ saddles to accommodate the beam tube, so there is no axial force on that subassembly. The friction lock of the preloaded interface between coil assembly and flux return is relied on to control the axial load on the top and bottom coil subassemblies. A problem is anticipated during the curing cool down, the Inconel blocks in the coil subassemblies will shrink less than the windings, reducing the vertical preload on the windings and reducing the friction lock. This problem is solved by using titanium for the blocks within the coil subassemblies as shown in Fig. 1. Titanium has a lower coefficient of expansion than either Inconel, the windings, or iron; so the vertical preload on the windings actually increases somewhat.

\section{E. Flux plate suppression of persistent magnetization fields}

A pair of magnetic steel flux plates is sandwiched between the top/bottom subassemblies and the central subassembly, as shown in Fig. 1. At injection field strength, the flux plate presents an unsaturated dipole boundary condition that suppresses sextupole and higher multipoles produced by the persistent current magnetization within the coils. Fig. 3 shows the calculated field distribution at injection $(0.5 \mathrm{~T})$, in which the re-arrangement of field lines in the flux plate is clearly evident.

This benefit is important for the use of high-performance $\mathrm{Nb}_{3} \mathrm{Sn}$, with a larger magnetization than $\mathrm{NbTi}$.

\section{CONSTRUCTION STATUS OF MODEL DIPOLES}

A first model dipole of this design is currently under construction, in which a single top coil subassembly module is being fabricated using cables of ITER $\mathrm{Nb}_{3} \mathrm{Sn}$ strand. It will be mounted in the flux return assembly with steel spacers replacing the other coil subassembly modules in order to independently test it cold. This module will be the final check of the fixtures and processing. All parts for these outer windings are fabricated including springs. All fixtures, winding fixture (complete), heat treat furnace (re-test), impregnation/cure vessel and heating system (additional lift fixturing), bladder pressurization and eutectic melting heater/reservoir system (final test), and the insulation processing (washing, drying, and resizing) line (complete) are either complete and tested or tested and being modified as needed before final tests.

Thereafter a succession of model dipoles will be built and tested: first a similar single-subassembly model using highperformance $\mathrm{Nb}_{3} \mathrm{Sn}$ strand, then a pair of such subassemblies, and finally the full dipole shown in Fig. 1. The completion and testing of the first module will occur next year.

\section{ACKNOWLEDGEMENT}

It is a pleasure to acknowledge the many design elements that have been adopted from our colleagues, including $\mathrm{W}$. Sampson, C. Taylor, and our collaborators in AFRD's Supercon Program at LBNL.

\section{REFERENCES}

[1] J.M. Van Ort, "Critical current degradation in $\mathrm{Nb}_{3} \mathrm{Sn}$ superconductors in accelerator magnets”, Ph.D. thesis, Universiteit Twente (2000).

[2] A.P. Verweij, "Electrodynamics of superconducting cables in accelerator magnets", Ph.D. thesis, Universiteit Twente (1995).

[3] B. Benjegerdes, et al., "Fabrication and Test of $\mathrm{Nb}_{3} \mathrm{Sn}$ Racetrack Coils at High Fields”, IEEE Trans. Appl. Supercond., 11, pp 2164-7 (2001).

[4] N. Diaczenko et al., "Stress management in high-field dipoles", Proc. 1997 Part.Accel.Conf, Vancouver, 1997, p. 3443-5. IEEE 97CH36167

[5] C. Battle et al., "Optimized block-coil dipoles for future hadron colliders”, IEEE Trans. Appl. Supercond., 10, pp 334-7(2000).

[6] R. Blackburn et al., "12 Tesla hybrid block-coil dipole for future hadron colliders”, IEEE Trans. Appl. Supercond.,l1, pp 2264-7 (2001).

[7] C. Battle et al., "Testing of TAMU1: A Single-aperture block-coil dipole”, Proc. 2001 Part. Accel. Conf., Chicago, 2001, pp 3642-4,IEEE $01 \mathrm{CH} 37268$. 
[8] A. McInturff, P. McIntyre, A. Sattarov, "Microbore dipole for future hadron colliders”, Proc. Snowmass 2001, T202, Snowmass, CO (2001). 